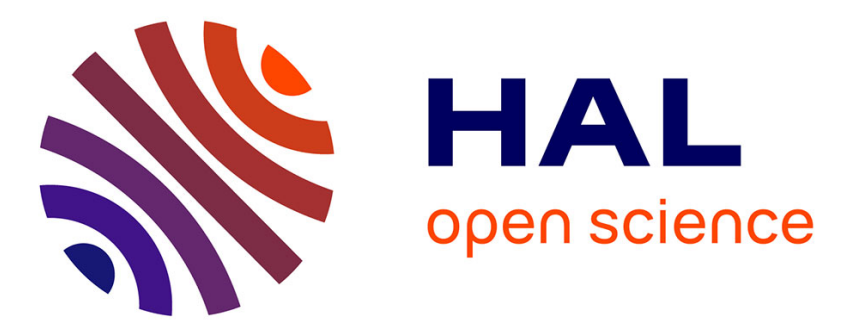

\title{
Parameter estimation of permanent magnet stepper motors without position or velocity sensors
}

\author{
Romain Delpoux, Marc Bodson, Thierry Floquet
}

\section{To cite this version:}

Romain Delpoux, Marc Bodson, Thierry Floquet. Parameter estimation of permanent magnet stepper motors without position or velocity sensors. American Control Conference, Jun 2012, Montreal, Canada. hal-00686349

\section{HAL Id: hal-00686349 \\ https://hal.inria.fr/hal-00686349}

Submitted on 10 Apr 2012

HAL is a multi-disciplinary open access archive for the deposit and dissemination of scientific research documents, whether they are published or not. The documents may come from teaching and research institutions in France or abroad, or from public or private research centers.
L'archive ouverte pluridisciplinaire HAL, est destinée au dépôt et à la diffusion de documents scientifiques de niveau recherche, publiés ou non, émanant des établissements d'enseignement et de recherche français ou étrangers, des laboratoires publics ou privés. 


\title{
Parameter estimation of permanent magnet stepper motors without position or velocity sensors
}

\author{
Romain Delpoux, Marc Bodson and Thierry Floquet
}

\begin{abstract}
The paper presents a new sensorless parameter identification method for permanent magnet stepper motors. Current sensors are assumed available, but position and velocity sensors are not. Data is obtained with open-loop voltage commands at multiple speeds. A new reference frame is proposed that presents advantages similar to the standard $d-q$ frame, but without the need for a position sensor. The method exploits carefully derived linear parameterizations and a least-squares algorithm. In one case, overparameterization is resolved using elimination theory. Overall, the parameters identified using the new procedure are found to be very close to those obtained with position sensors. The approach is potentially applicable to other types of synchronous motors as well.
\end{abstract}

Index Terms-Permanent magnet stepper motors, synchronous motors, sensorless systems, parameter identification, elimination theory

\section{INTRODUCTION}

Permanent Magnet Stepper Motors (PMSM's) are widely used in industry for position control, especially in manufacturing applications. PMSM's are more robust than brush DC motors and produce high torque per volume. They are often controlled in open-loop, although the potential loss of synchronism limits operation away from resonances and from high acceleration trajectories. These problems can be resolved by using closed-loop control methods with position sensors of sufficient precision. Recent research has focussed on whether the performance of closed-loop control methods could be achieved using sensorless systems. In this case, sensorless refers to systems that do not have position sensors, although current sensors are still assumed to be available.

Current sensors can reconstruct the position of the rotor through the induced back-emf voltage at non-zero speeds [4], [7] and [13]. For such methods to succeed, the model of the motor and its parameters have to be well known, which brings to the forefront the question of parameter identification without position or velocity sensors. The estimation of PMSM parameters was studied in [1], [8], and [10], but with rotor position information. Position sensorless identification was applied using special signals at standstill or under load condition in [11], but for the identification of the $d$ and $q$ reactances only. Other methods to identify motor parameters online include [2], [9], but [2] only provides

R. Delpoux and T. Floquet are with Laboratoire d'Automatique, Génie Informatique et Signal (CNRS FRE 3303), Ecole Centrale de Lille, 59651 Villeneuve d'Ascq cedex, France and Team Non-A, INRIA Lille Nord Europe, France romain. delpouxdec-lille.fr and thierry.floquet@ec-lille.fr

M. Bodson is with the Power Electronics and Automation Research Laboratory (PEARL), Department of Electrical and Computeur Engineering, University of Utah, Salt Lake City, UT 84112, USA bodsoneeng. utah.edu simulation results and in [9], only the stator resistance and the back EMF constant are identified. In [5], [6], [16], parameter identification is realized in the $d-q$ frame, where the position needed for the $d-q$ transformation is estimated using identified parameters. This type of structure may be successful in practice, but guarantees of stability and convergence are absent, because parameter estimation depends on position estimation and vice-versa.

This paper presents a new method for the identification of the parameters of a PMSM without a position or velocity sensor. Compared to existing approaches, the new method has the advantages of: identifying all of the electrical parameters as well as the mechanical parameters, deriving identification algorithms that are guaranteed to converge, validating the analytical results with experimental data, and comparing the results obtained without position sensors to those obtained with sensors and using comparable methods. Another contribution of the paper is the presentation and use of a new reference frame that is advantageous for sensorless applications. Experiments were performed using a test bench available at the LAGIS laboratory at École Centrale de Lille. The article is divided into three parts. Section II presents the model of the PMSM in three different reference frames. In Section III, an identification procedure is developed for systems with position and velocity sensors, and it is tested experimentally. The last section IV presents the new identification procedure and the results obtained experimentally.

\section{Model OF THE PMSM}

In this section, the model of the PMSM is presented in three different frames, including a new frame that is particularly useful for sensorless applications.

\section{A. Model in the phase variables $(a-b)$}

Equations (1) give the standard PMSM model in the phase (or winding) variables

$$
\left\{\begin{aligned}
L \frac{d i_{a}}{d t}= & v_{a}-R i_{a}+K \Omega \sin (N \theta) \\
L \frac{d i_{b}}{d t}= & v_{b}-R i_{b}-K \Omega \cos (N \theta) \\
J \frac{d \Omega}{d t}= & K\left(i_{b} \cos (N \theta)-i_{a} \sin (N \theta)\right) \\
& -f_{v} \Omega-C_{r} \operatorname{sgn}(\Omega)
\end{aligned}\right.
$$

where $v_{a}$ and $v_{b}$ are the voltages applied to the two phases of the PMSM, $i_{a}$ and $i_{b}$ are the two phase currents, $L$ is the inductance of a phase winding, $R$ is the resistance of a phase winding, $K$ is the back-EMF constant (and also the torque 
constant), $\theta$ is the angular position of the rotor, $\Omega=d \theta / d t$ is the angular velocity of the rotor, $N$ is the number of pole pairs (or rotor teeth), $J$ is the moment of inertia of the rotor (including the load), $f_{v}$ is the coefficient of viscous friction, and $C_{r}$ is the coefficient of Coulomb friction.

\section{B. Model in the rotating frame $(d-q)$}

The phase model can be transformed using Park's transformation [12],

$$
\begin{aligned}
{\left[i_{d}, i_{q}\right]^{T} } & =M_{p}\left[i_{a}, i_{b}\right]^{T} \\
{\left[v_{d}, v_{q}\right]^{T} } & =M_{p}\left[v_{a}, v_{b}\right]^{T}
\end{aligned}
$$

where

$$
M_{p}=\left[\begin{array}{cc}
\cos (N \theta) & \sin (N \theta) \\
-\sin (N \theta) & \cos (N \theta)
\end{array}\right]
$$

Using this change of coordinates, the system (1) is transformed into the so-called $d-q$ model

$$
\left\{\begin{array}{l}
L \frac{d i_{d}}{d t}=v_{d}-R i_{d}+N L \Omega i_{q} \\
L \frac{d i_{q}}{d t}=v_{q}-R i_{q}-N L \Omega i_{d}-K \Omega \\
J \frac{d \Omega}{d t}=K i_{q}-f_{v} \Omega-C_{r} \operatorname{sgn}(\Omega)
\end{array}\right.
$$

The $d-q$ transformation is commonly used for PMSM's (and synchronous motors in general), because it results in constant voltages and currents at constant speed (instead of the highfrequency phase variables). Also, the model highlights the role of the quadrature current $i_{q}$ in determining the torque. However, the $d-q$ transformation is based on the position $\theta$, which is not directly available in sensorless applications.

\section{$C$. Model in the rotating reference frame $(f-g)$}

To overcome the problems caused by the use of the position in the $d-q$ frame, a different reference frame is proposed that uses a reference position instead of the real position. The transformation is expressed in matrix form as

$$
\begin{aligned}
{\left[i_{f}, i_{g}\right]^{T} } & =M_{r}\left[i_{a}, i_{b}\right]^{T} \\
{\left[v_{f}, v_{g}\right]^{T} } & =M_{r}\left[v_{a}, v_{b}\right]^{T}
\end{aligned}
$$

where

$$
M_{r}=\left[\begin{array}{cc}
\cos \left(N \theta_{r}\right) & \sin \left(N \theta_{r}\right) \\
-\sin \left(N \theta_{r}\right) & \cos \left(N \theta_{r}\right)
\end{array}\right]
$$

and $\theta_{r}$ is an arbitrary reference position.

The PMSM model in the transformed variables is

$$
\left\{\begin{aligned}
L \frac{d i_{f}}{d t}= & v_{f}-R i_{f}-K \Omega \sin \left(N\left(\theta_{r}-\theta\right)\right) \\
& +L N \Omega_{r} i_{g} \\
L \frac{d i_{g}}{d t}= & v_{g}-R i_{g}-K \Omega \cos \left(N\left(\theta_{r}-\theta\right)\right) \\
& -L N \Omega_{r} i_{f} \\
J \frac{d \Omega}{d t}= & K\left(i_{f} \sin \left(N\left(\theta_{r}-\theta\right)\right)+\right. \\
& \left.i_{g} \cos \left(N\left(\theta_{r}-\theta\right)\right)\right) \\
& -f_{v} \Omega-C_{r} \operatorname{sgn}(\Omega)
\end{aligned}\right.
$$

where $\Omega_{r}=d \theta_{r} / d t$. The $f-g$ frame is potentially useful in two ways. First, $\theta_{r}$ may be defined as the reference position that the motor is supposed to track. For example, an openloop voltage control strategy consists in setting $v_{f}=V_{0}$, $v_{g}=0$. Second, $\theta_{r}$ may be defined as an estimate of $\theta$, using a procedure to be determined. Then, the $f-g$ model approximates the $d-q$ model, with the advantage that it is valid and computable even if $\theta_{r}$ is not exactly equal to $\theta$. We will consider the problem of sensorless parameter identification in the $f-g$ frame, setting $\theta_{r}$ as a reference position. In order to provide a basis for comparison, however, we first discuss identification in the $d-q$ frame using a position sensor.

\section{PARAMETER IDENTIFICATION USING A POSITION SENSOR}

If a position sensor is available, the $d-q$ frame is attractive to perform identification. Most variables can be estimated using steady-state measurements at constant speed and, because the $d-q$ variables can be averaged over batches of data, good robustness to noise and unmodeled effects can be obtained. Such effects include the detent torque, biases in the voltages, measurement biases in the currents, and amplifier distortions. The core of the identification procedure is a least-squares algorithm [1]. Consider an equation

$$
y[n]=W^{T}[n] P_{n o m}
$$

where $y$ is the output vector, $W$ is the regressor matrix, and $P_{\text {nom }}$ is the nominal (unknown) parameter vector. Given measurements of $y$ and $W$, the objective is to determine $P$, an estimate of the nominal parameter vector $P_{n o m}$. The estimated parameters are obtained by least-squares algorithm.

\section{A. Parameter estimation using steady-state measurements}

At steady-state, the model in the $d-q$ frame gives

$$
\left[\begin{array}{l}
v_{d} \\
v_{q}
\end{array}\right]=\left[\begin{array}{ccc}
i_{d} & -N \Omega i_{q} & 0 \\
i_{q} & N \Omega i_{d} & \Omega
\end{array}\right]\left[\begin{array}{c}
R \\
L \\
K
\end{array}\right]
$$

which is linear in the parameters, so that the least-squares algorithm can be used with

$$
\begin{gathered}
y[n]=\left[\begin{array}{c}
v_{d}[n] \\
v_{q}[n]
\end{array}\right] \\
W^{T}[n]=\left[\begin{array}{ccc}
i_{d}[n] & -N \Omega[n] i_{q}[n] & 0 \\
i_{q}[n] & N \Omega[n] i_{d}[n] & \Omega[n]
\end{array}\right] \\
P_{n o m}=\left[\begin{array}{ccc}
R & L & K
\end{array}\right]^{T}
\end{gathered}
$$

To identify $R, L$, and $K$, experiments are performed with various voltages $v_{d}$ and $v_{q}$, which are transformed in the phase variables using (3) and applied to the motor. The currents $i_{d}$ and $i_{q}$ are obtained using (2). Once the velocity is (approximately) constant, the average values of the currents and of the velocity are computed over a small time interval, yielding one data point for the least-squares algorithm. Various values of $v_{d}$ and $v_{q}$ are applied to the motor to obtain velocities covering the whole velocity range. Multiple data points are thereby obtained for the least-squares algorithm, from which estimates $\hat{R}, \hat{L}$, and $\hat{K}$ can be computed. 

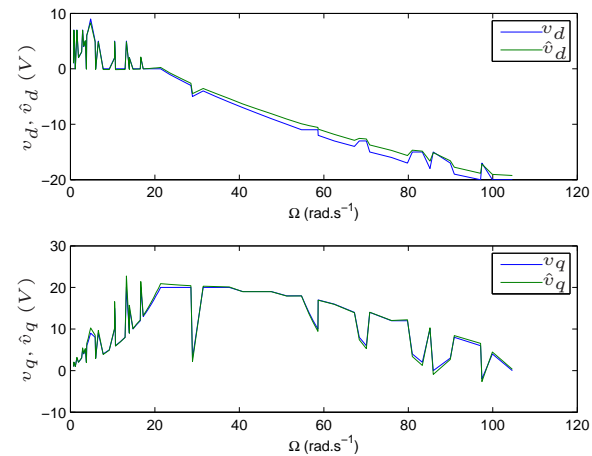

Fig. 1. Representation of the identification of the electrical parameters in the $d-q$ frame

The estimate of $K$ obtained in the first step is used in the mechanical equation in order to identify two more parameters using

$$
\left[\hat{K} i_{q}\right]=\left[\begin{array}{ll}
\Omega & \operatorname{sgn}(\Omega)
\end{array}\right]\left[\begin{array}{l}
f_{v} \\
C_{r}
\end{array}\right]
$$

Again, equation (15) is linear in the parameters, so that the least-squares algorithm can be used to obtain estimates of $f_{v}$ and $C_{r}$.

The results of identification using this two-step procedure are shown in Table I. Fig. 1 shows the output vector from (11) as a function of $\Omega$, as well as the fit from the leastsquares algorithm (i.e., $W^{T}[n] P$, where $P$ is the leastsquares estimate of the parameters). The components of the output vector are the voltages $v_{d}$ and $v_{q}$, whose jagged appearance is due to the fact that multiple values of the voltages can yield the same velocity, and multiple values were deliberately applied to increase the richness of the data. The match of the least-squares fit with the data on the figure is found to be very good.

\section{B. Parameter estimation of inertia using transient measure- ments}

The inertia parameter does not affect the dynamics in steadystate, and is the only parameter that requires the measurement of a transient response. Using the estimates $\hat{K}, \hat{f}_{v}$, and $\hat{C}_{r}$ obtained in steady-state, the mechanical equation in the $d-q$ frame gives an equation

$$
\left[\hat{K} i_{q}-\hat{f}_{v} \Omega-\hat{C}_{r} \operatorname{sgn}(\Omega)\right]=[\dot{\Omega}][J]
$$

\begin{tabular}{|c|c|c|}
\hline \hline Parameters & $d-q$ frame & $f-g$ frame \\
\hline$R(\Omega)$ & 2.86 & 2.86 \\
$L(m H)$ & 10.2 & 10.4 \\
$K\left(N m . A^{-1}\right)$ & 0.26 & 0.27 \\
\hline$f_{v}(N m s / r a d)$ & $2.37 .10^{-4}$ & $2.69 .10^{-4}$ \\
$C_{r}$ & 0.0752 & 0.0742 \\
\hline$J\left(k g . m^{2}\right)$ & $3.18 .10^{-4}$ & $3.13 .10^{-4}$ \\
\hline \hline
\end{tabular}

TABLE I

RESULTS OF THE PARAMETER ESTIMATION.

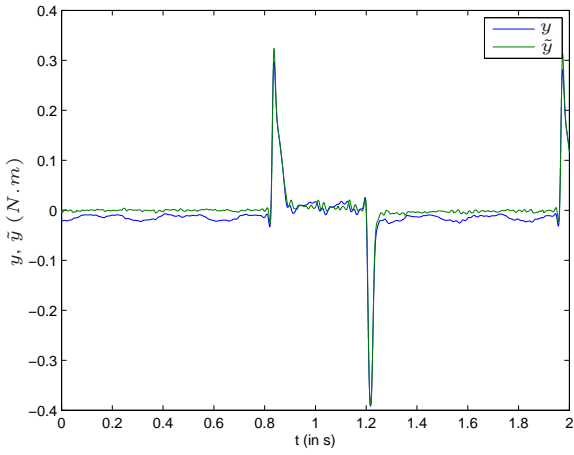

Fig. 2. Inertia estimation in the $d-q$ frame

which is linear in the parameter $J$ to be estimated. A large step in voltage is applied to produce a large acceleration $d \Omega / d t$, which is necessary to obtain a reliable estimate in the presence of unmodeled effects in the mechanical equation.

The angular acceleration can be reconstructed from the velocity using the difference equationThe resulting signal is low-pass filtered to reduce noise (in the experiments, a third-order Butterworth with cut-off frequency at $500 \mathrm{~Hz}$ was used). Using the Matlab function filtfilt, then no delays are introduced. Note that, in general, velocity may have to be reconstructed from position using a similar procedure. However, the testbed used in the experiments of this paper included a tachometer as well as an encoder, so that it was not necessary to do so.

When step changes in voltages are applied, the velocity changes rapidly, yielding an acceleration profile such as shown in Fig. 2. The response exhibits spikes, which are well matched by the least-squares fit. The identification results are shown in Table I. Using the $d-q$ frame, all the parameters of the model have been identified. The estimates provide a reference to compare with in the sensorless identification section presented next.

\section{SENSORLESS PARAMETER IDENTIFICATION}

The $d-q$ model is beneficial for identification compared to the $a-b$ model, because it makes it possible to average data over batches at constant speed. However, its drawback is that it cannot be used without a position sensor. To derive an algorithm for position-sensorless identification, the $f-g$ transformation is advantageous.

Identification experiments can be performed in the $f-g$ frame similarly to the $d-q$ frame, specifically with constant voltages $v_{f}$ and $v_{g}$ applied at constant speed. However, as opposed to the $d-q$ frame, the PMSM cannot be operated for all speeds in this manner. There are regions of the speed range where the PMSM loses synchronism due to resonances. Acceleration is also limited.

When the PMSM is controlled in the $f-g$ frame, it can be assumed that $\Omega_{r} \simeq \Omega$ as long as the motor keeps synchronism. This condition can be verified without a sensor because loss of synchronism results in a stalled motor and/or 
considerable vibrations. Further, even if $\Omega$ oscillates around $\Omega_{r}$ due to disturbance torques, averaging of the data over periods of time removes the effect of these oscillations, so that one can assume that $\Omega_{r}=\Omega$.

In order to maximize the information content of the data, experiments were performed using the following proportional controller in the rotating reference frame

$$
v_{f}=\max \left(k\left(I_{\max }-I\right), V_{\max }\right), v_{g}=0
$$

where $I=i_{a}^{2}+i_{b}^{2}$ is the peak current, $I_{\max }$ is the current limit, $V_{\max }$ is the voltage limit, and $k$ is an adjustable gain. This control law was used to maximize the currents and voltages while respecting the limits. The control law was applied with multiple values of $\Omega_{r}$, which were slowly increased from $\Omega_{r}=0$. Fig. 3 shows the average speeds and the speed references for the experiments of the paper. The figure shows that the set of average speeds indeed followed the reference speeds when the control (17) was used. On the figure, one can see a gap caused by resonances between 14 and $28 \mathrm{rad} / \mathrm{s}$. However, speeds well above the resonant region could be reached.

\section{A. Parameter estimation using steady-state measurements}

As for the $d-q$ identification, almost all the parameters can be estimated with steady-state experiments in the $f-g$ frame, leaving the identification of the inertia to a well-designed transient experiment. In steady-state, the variables in the $f-g$ frame are related through

$$
\begin{aligned}
K \Omega \sin \left(N\left(\theta_{r}-\theta\right)\right)= & v_{f}-R i_{f}+L N \Omega_{r} i_{g} \\
K \Omega \cos \left(N\left(\theta_{r}-\theta\right)\right)= & v_{g}-R i_{g}-L N \Omega_{r} i_{f} \\
0= & K\left(i_{f} \sin \left(N\left(\theta_{r}-\theta\right)\right)\right. \\
& \left.+i_{g} \cos \left(N\left(\theta_{r}-\theta\right)\right)\right) \\
& -f_{v} \Omega-C_{r} \operatorname{sign}(\Omega)
\end{aligned}
$$

Although linear parameterizations are apparent as in the $d-q$ frame, they cannot be used due to the unknown rotor position. A different procedure must be used. Substituting (18) and (19) in (20), one finds

$$
v_{f} i_{f}+v_{g} i_{g}=R\left(i_{f}^{2}+i_{g}^{2}\right)+f_{v} \Omega^{2}+C_{r}|\Omega|
$$

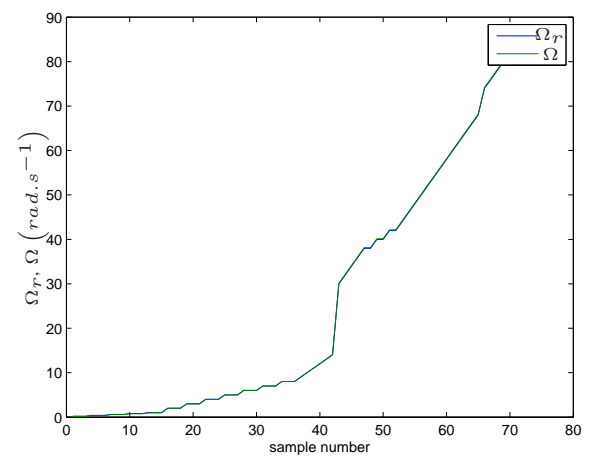

Fig. 3. Speed tracking in experiment

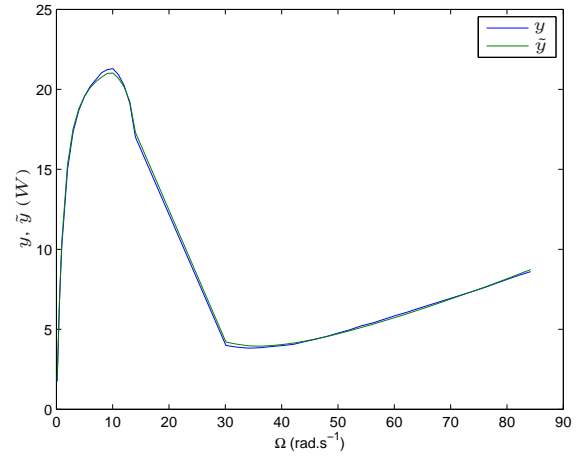

Fig. 4. Representation of the identification of the mechanical parameters in the $f-g$ frame

The equation is linear with respect to the parameters with

$$
\begin{aligned}
& y[n]=v_{f}[n] i_{f}[n]+v_{g}[n] i_{g}[n] \\
& W^{T}[n]=\left[\begin{array}{lll}
i_{f}[n]^{2}+i_{g}[n]^{2} & \Omega[n]^{2} & |\Omega[n]|
\end{array}\right] \\
& P_{\text {nom }}=\left[\begin{array}{lll}
R & f_{v} & C_{r}
\end{array}\right]^{T}
\end{aligned}
$$

so that a least-squares algorithm is applicable.

The results for the identification using equation (21) are shown in Table I. The estimated parameters are close to the parameters estimated in the $d-q$ frame using a position sensor. Fig. 4 shows the output variable, which is the power absorbed by the motor. The least-squares fit is excellent.

For the identification of $L$ and $K$, consider $(18)^{2}+(19)^{2}$, which yields

$$
\begin{aligned}
K^{2} \Omega^{2}= & v_{f}^{2}+v_{g}^{2}+R^{2}\left(i_{f}^{2}+i_{g}^{2}\right)-R\left(2 v_{f} i_{f}+2 v_{g} i_{g}\right)+ \\
& L^{2}\left(N^{2} \Omega_{r}^{2}\left(i_{f}^{2}+i_{g}^{2}\right)\right)+L\left(2 N \Omega_{r}\left(v_{f} i_{g}-v_{f} i_{g}\right)\right)
\end{aligned}
$$

The relationship (25) can be written in the form of a linear equation using $\hat{R}$, the estimate of $R$ obtained in the first step (another option would be to measure the resistance with a DC voltage), so that

$$
y[n]=W^{T}[n] P_{n o m}
$$

with

$$
\begin{gathered}
y[n]=\left[\begin{array}{l}
v_{f}[n]^{2}+v_{g}[n]^{2}-2 \hat{R}\left(v_{f}[n] i_{f}[n]\right. \\
\left.+v_{g}[n] i_{g}[n]\right)-\hat{R}^{2}\left(i_{f}[n]^{2}+i_{g}[n]^{2}\right)
\end{array}\right. \\
W[n]=\left[\begin{array}{c}
-2 N \Omega_{r}\left(v_{f}[n] i_{g}[n]-v_{g}[n] i_{f}[n]\right) \\
-N^{2} \Omega_{r}[n]^{2}\left(i_{f}[n]^{2}+i_{g}[n]^{2}\right) \\
\Omega[n]^{2}
\end{array}\right] \\
P_{n o m}=\left[\begin{array}{lll}
L & L^{2} & K^{2}
\end{array}\right]=\left[\begin{array}{ll}
p_{n o m, 1} & p_{n o m, 2}
\end{array}\right]
\end{gathered}
$$

The system (26) is linear in the parameters, but it is overparametrized since

$$
p_{\text {nom }, 2}=p_{\text {nom }, 1}^{2}
$$


The overparameterization can be handled using techniques described in [3], [14] and [15], where the problem is reformulated as a least-squares minimization objective

$$
E^{2}(P)=\sum_{n=1}^{N}|y(n)-W(n) P|^{2}
$$

subject to the contraint $p_{2}=p_{1}^{2}$. Define

$$
P_{m} \triangleq\left[\begin{array}{ll}
p_{1} & p_{3}
\end{array}\right]^{T}
$$

and

$$
E^{2}\left(P_{m}\right)=\sum_{n=1}^{N}|y(n)-W(n) P|_{p_{2}=p_{1}^{2}}^{2}
$$

The value of $P_{m}$ that minimizes the residual error $E^{2}\left(P_{m}\right)$ is obtained by solving the two equations

$$
\pi_{1}\left(P_{m}\right) \triangleq \frac{\partial E^{2}\left(P_{m}\right)}{\partial p_{1}}=0, \pi_{2}\left(P_{m}\right) \triangleq \frac{\partial E^{2}\left(P_{m}\right)}{\partial p_{3}}=0
$$

$\pi_{1}\left(P_{m}\right)$ and $\pi_{2}\left(P_{m}\right)$ are polynomials in the parameters $p_{1}$ and $p_{3}$. In general, such system of equations can be solved using resultants. Thus, one computes the resultant

$$
r\left(p_{1}\right) \triangleq \operatorname{Res}\left(\pi_{1}, \pi_{2}, p_{3}\right)
$$

to eliminate $p_{3} . r\left(p_{1}\right)$ is a polynomial in $p_{1}$ (only), whose roots are substituted in $\pi_{1}\left(P_{m}\right)$, which is then a polynomial in $p_{3}$ only. For each root $p_{1 i}$, solutions $\left(p_{1 i}, p_{3 i j}\right)$ of $\pi_{1}\left(P_{m}\right)$ are then obtained. The solutions that also satisfy $\pi_{2}\left(P_{m}\right)$ are the possible solutions of the least-squares problem. In the experiments of this paper, the computations were carried out symbolically using Maple.

The results of the identification for $L$ and $K$ are shown Table I. The estimated parameters are close to those obtained in the $d-q$ frame using a position sensor. Moreover, Fig. 5 shows the output variable from (26) as a function of $\Omega$. The least-squares fit is very good.

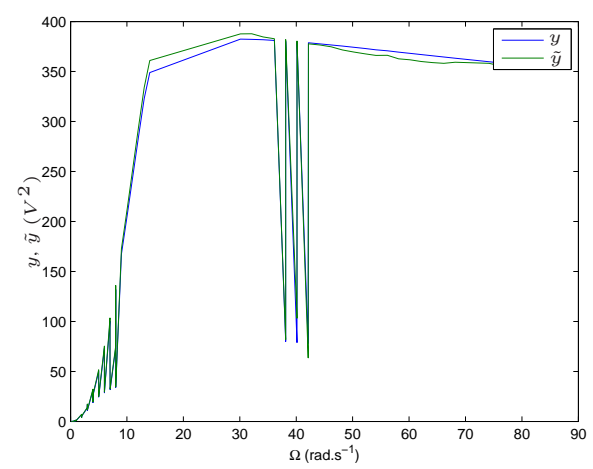

Fig. 5. Representation of the identification of the electrical parameters in the $f-g$ frame

\section{B. Parameter estimation of inertia using transient measure-} ments

Without a position sensor, the torque produced by the motor cannot be directly computed from the currents. In order to circumvent this problem, the technique discussed in this section is based on a computation of power rather than torque. Specifically, the electrical power converted to mechanical power is

$$
\begin{aligned}
P_{e m} & \triangleq v_{a} i_{a}+v_{b} i_{b}-R i_{a}^{2}-R i_{b}^{2}-\frac{L}{2} \frac{d}{d t}\left(i_{a}^{2}+i_{b}^{2}\right) \\
& =K \Omega\left(-i_{a} \sin (N \theta)+i_{b} \cos (N \theta)\right)
\end{aligned}
$$

Using the mechanical equation of the model, one also has

$$
P_{e m}=P_{k i n}+P_{f}
$$

where $P_{k i n}$ is the kinetic power, i.e., the variation in kinetic energy

$$
P_{k i n} \triangleq J \Omega \dot{\Omega}
$$

while $P_{f}$ is the power lost to friction

$$
P_{f}=f_{v} \Omega^{2}+C_{r}|\Omega|
$$

The inertia can therefore be computed using a least-squares algorithm or, simply

$$
\hat{J}=\frac{P_{e m}-P_{f}}{\Omega(d \Omega / d t)}
$$

In order to reduce the effect of noise and unmodeled dynamics, the inertia experiment is carried out as follows. With constant $v_{f}$ and $v_{g}$, the motor is operated at some arbitrary reference velocity $\Omega_{r}=\Omega_{1}$, and data is taken for a period $\left[t_{0}, t_{1}\right]$. The friction power $P_{f, 1}$ is computed as the average of $P_{e m}$ over $\left[t_{0}, t_{1}\right]$ (since $d \Omega / d t=0$ ). Then, the reference velocity is rapidly increased using

$$
\Omega_{r}(t)=\sqrt{\Omega_{1}^{2}+2 c\left(t-t_{1}\right)}
$$

This reference profile is such that

$$
\Omega_{r} \dot{\Omega}_{r}=c
$$

and $c$ is adjusted experimentally as large as possible to maximize the information content of the data. The acceleration continues until some time $t_{2}$ where an arbitrary reference velocity $\Omega_{2}$ is reached. Then, a third batch of data is collected at constant speed $\Omega_{2}$ for a period $\left[t_{2}, t_{3}\right]$, and the friction power $P_{f, 2}$ is computed as the average of $P_{\text {em }}$ over $\left[t_{2}, t_{3}\right]$.

The velocity and the reference velocity for such an experiment are shown Fig. 6. One finds that tracking is only approximate, but the collection of data over periods of time enables the hypothesis that $\Omega_{r} \cong \Omega$. The inertia estimate is then computed using

$$
\hat{J}=A V G\left[\left(P_{e m}-\hat{P}_{f}\right) / c\right]
$$

where the average is taken over $\left[t_{1}, t_{2}\right]$ and $\hat{P}_{f}$ is an estimate of the friction. $\hat{P}_{f}$ can be obtained using the estimated coefficients of friction obtained in the previous 
section. Alternatively, the experiments discussed here used the interpolation formula

$$
\hat{P}_{f}=P_{f, 1}+\left(P_{f, 2}-P_{f, 1}\right)\left(t-t_{1}\right) /\left(t_{2}-t_{1}\right)
$$

Also, the contribution of the inductive term in $P_{e m}$ was neglected in the computations.

The experiment realized for the estimation of the inertia is plotted Fig. 6. The estimate of inertia was further refined by repeating the experiment with different velocities $\Omega_{1}$ and $\Omega_{2}$, and with different values of $c$. The resulting estimate is shown in Table I, and matches closely the value estimated with a position sensor.

\section{Conclusions}

There has been considerable interest in developing sensorless control methods for synchronous motors, and permanent magnet stepper motors in particular. The objective is to replace position and velocity sensors with less costly and more reliable current sensors (which are often present anyway). Sensorless methods rely on the knowledge of a model and of its parameters, which raises the question of how these parameters can be determined without position sensors.

In this paper, a new approach was proposed for the estimation of the parameters of PMSM's using current sensors only. Data was collected with the motor operated in open-loop, which is always possible with PMSM's, although certain speed ranges may be prohibited and accelerations may be limited. Although open-loop tracking of a speed reference may not be satisfactory from a control perspective, the paper showed that the information gathered was sufficient to determine all the parameters of the model. Results were close to those that were obtained with a position sensor.

A special frame of reference was introduced based on the reference position. It presented the advantage of resulting in constant voltages and currents at constant speed, yet without the need for a position sensor. The reference frame may be useful for sensorless control as well. An interesting possibility is to have $\theta_{r}$ be an estimate of the position $\theta$, using an observer to be derived using the $f-g$ model.

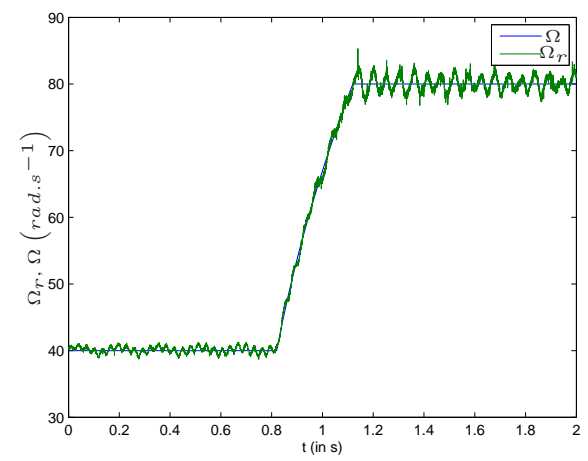

Fig. 6. Inertia identification experiment

\section{ACKNOWLDEGMENTS}

This work was supported by Ministry of Higher Education and Research, Nord-Pas-de-Calais Regional Council and FEDER through the "Contrat de Projets Etat Region (CPER) 2007-2013". Marc Bodson acknowledges the support of the École Centrale de Lille through a visiting professorship.

\section{REFERENCES}

[1] A.J. Blauch, M. Bodson, and J. Chiasson. High-speed parameter estimation of stepper motors. Control Systems Technology, IEEE Transactions on, 1(4):270 -279, dec 1993.

[2] S. Bolognani, M. Zigliotto, and K. Unterkofler. On-line parameter commissioning in sensorless pmsm drives. In Industrial Electronics, 1997. ISIE '97., Proceedings of the IEEE International Symposium on, volume 2, pages $480-484$ vol.2, jul 1997.

[3] J. Chiasson and A. Oteafy. Elimination theory for nonlinear parameter estimation. In Jean Levine and Philippe Mullhaupt, editors, Advances in the Theory of Control, Signals and Systems with Physical Modeling, volume 407 of Lecture Notes in Control and Information Sciences, pages 65-75. Springer Berlin / Heidelberg, 2011.

[4] C. Fiter, T. Floquet, and J. Rudolph. Sensorless Control of a Stepper Motor Based on Higher Order Sliding Modes. In 8th IFAC Symposium on Nonlinear Control Systems (NOLCOS 2010), pages -, Bologna, Italy, 2010.

[5] S. Ichikawa, M. Tomita, S. Doki, and S. Okuma. Sensorless control for all types of synchronous motors using an on-line parameter identification. In Industrial Electronics Society, 2004. IECON 2004. 30th Annual Conference of IEEE, volume 1, pages 975 - 980 Vol. 1, nov. 2004.

[6] S. Ichikawa, M. Tomita, S. Doki, and S. Okuma. Sensorless control of permanent-magnet synchronous motors using online parameter identification based on system identification theory. Industrial Electronics, IEEE Transactions on, 53(2):363 - 372, april 2006.

[7] J.P. Johnson, M. Ehsani, and Y. Guzelgunler. Review of sensorless methods for brushless dc. In Industry Applications Conference, 1999. Thirty-Fourth IAS Annual Meeting. Conference Record of the 1999 IEEE, volume 1, pages $143-150$ vol.1, 1999.

[8] H. Kim and R.D. Lorenz. Improved current regulators for ipm machine drives using on-line parameter estimation. In Industry Applications Conference, 2002. 37th IAS Annual Meeting. Conference Record of the, volume 1, pages $86-91 \mathrm{vol} .1,2002$.

[9] K.W. Lee, D.H. Jung, and I.J. Ha. An online identification method for both stator resistance and back-emf coefficient of pmsms without rotational transducers. Industrial Electronics, IEEE Transactions on, 51(2):507 - 510, april 2004.

[10] B. Nahid Mobarakeh, F. Meibody-Tabar, and F.M. Sargos. On-line identification of pmsm electrical parameters based on decoupling control. In Industry Applications Conference, 2001. Thirty-Sixth IAS Annual Meeting. Conference Record of the 2001 IEEE, volume 1, pages $266-273$ vol.1, sep-4 oct 2001.

[11] H.-P. Nee, L. Lefevre, P. Thelin, and J. Soulard. Determination of d and $\mathrm{q}$ reactances of permanent-magnet synchronous motors without measurements of the rotor position. Industry Applications, IEEE Transactions on, 36(5):1330 -1335, sep/oct 2000.

[12] R. H. Park. Two-reaction theory of synchronous machines generalized method of analysis-part i. American Institute of Electrical Engineers, Transactions of the, 48(3):716 -727, july 1929.

[13] M. Schroedl. Sensorless control of permanent-magnet synchronous machines: An overview. In Proc. EPE-PEMC, 2004.

[14] K. Wang, M. Bodson, J. Chiasson, and L.M. Tolbert. Parameter identification of hammerstein models using elimination theory. In Decision and Control, 2005 and 2005 European Control Conference. CDC-ECC '05. 44th IEEE Conference on, pages $3444-3449$, dec. 2005.

[15] K Wang, J. Chiasson, M. Bodson, and L.M. Tolbert. A nonlinear leastsquares approach for identification of the induction motor parameters. Automatic Control, IEEE Transactions on, 50(10):1622 - 1628, oct. 2005.

[16] M. Yoshimi, M. Hasegawa, and K. Matsui. Parameter identification for ipmsm position sensorless control based on unknown input observer. In Industrial Electronics Applications (ISIEA), 2010 IEEE Symposium on, pages $383-388$, oct. 2010. 